JAMA Ophthalmol. 2016 September 01; 134(9): 1040. doi:10.1001/jamaophthalmol.2016.2017.

\title{
Optical Coherence Tomography in High Myopia
}

\author{
Joel S. Schuman, MD \\ Department of Ophthalmology, NYU Langone Medical Center, New York University School of \\ Medicine, New York
}

Optical coherence tomography (OCT) is a precise technology that can be used for interrogating tissue structure and function noninvasively, painlessly, and quickly using nearinfrared light. At its inception, OCT was typically a tool for assessing tissue thickness. Time-domain OCT is fast enough to scan a circle centered on the optic nerve head (circumpapillary) to assess retinal nerve fiber layer (RNFL) thickness. With the development of spectral-domain OCT, volumetric (3-dimensional) scanning becomes feasible.

With a circumpapillary scan, the RNFL thickness measurement is assumed to be relatively insensitive to ocular axial length. In myopia of up to approximately -10 diopters, the error would be negligible. That assumption is based on the size of the scanning circle, which increases in diameter with increasing distance from the OCT unit. Since the expanding circle enlarges a small amount, and the RNFL decreases with a function of approximately 1/radius with distance from the disc, no correction was thought to be needed, except in eyes with very high myopia.

The fallacy in that reasoning is that the retina actually does become thin in high myopia, an effect that is essentially doubled in eyes with high myopia with regard to OCT RNFL thickness measurements. The measurement circle is further from the center of the optic nerve, so the measured RNFL is thinner than it would be for a 3.4-mm circle centered on the optic nerve head, and the RNFL is thinner because it is stretched to cover the area of an elongated globe. This scenario results in a thinner than expected RNFL measurement on each of these 2 counts, with an additive effect confounding interpretation of OCT measurements in eyes with high myopia. The greater the axial myopia, the greater the artifactual (and actual) RNFL thinness.

High myopia is often the cause of what has been termed red disease, ${ }^{1}$ a pseudo-condition in which RNFL thickness measured by OCT is indicated as outside normal limits, for reasons noted above; physicians mistakenly interpret these findings as glaucoma. There are clues as to whether OCT results represent artifacts or true glaucomatous damage. In myopia-induced OCT artifacts, deviations from the normative database, indicated as red, are diffuse, and not

Corresponding Author: Joel S. Schuman, MD, Department of Ophthalmology, NYU Langone Medical Center, New York University School of Medicine, 462 First Ave, Ste NBV 5N3, New York, NY 10016 (joel.schuman@nyu.edu).

Conflict of Interest Disclosures: Dr Schuman has completed and submitted the ICMJE Form for Disclosure of Potential Conflicts of Interest. He reported receiving royalties for intellectual property licensed by Massachusetts Institute of Technology and Massachusetts Eye and Ear Infirmary to Zeiss. No other disclosures were reported.

Role of the Funder/Sponsor: The funding sources had no role in the preparation, review, or approval of the manuscript and decision to submit the manuscript for publication. 
limited to the superior and inferior arcuate areas expanding from the disc-regions typically associated with glaucoma. A second clue as to the verity of the OCT abnormalities is a normal visual field in the presence of a moderate to severe OCT abnormality, such as average RNFL thickness less than $75 \%$ of normal.

A mathematical solution may approximate true expected RNFL thickness values, but an empirical OCT normative database for eyes with high myopia, as suggested by Biswas et $\mathrm{al},{ }^{2}$ would address the problem directly. The use and utility of OCT are high. The time has come for commercial implementation of OCT normative databases for high myopia.

\section{Acknowledgments}

Funding/Support: This work was funded by grant R01-EY013178 from the National Institutes of Health and an unrestricted grant from Research to Prevent Blindness.

\section{References}

1. Chong GT, Lee RK. Glaucoma versus red disease: imaging and glaucoma diagnosis. Curr Opin Ophthalmol. 2012; 23(2):79-88. [PubMed: 22262083]

2. Biswas S, Lin C, Leung CKS. Evaluation of a myopic normative database for analysis of retinal nerve fiber layer thickness [published online July 21, 2016]. JAMA Ophthalmol. 